Planetary Systems in the Universe - Observation, Formation and Evolution

Proceedings IAU Symposium No. 202, (C2004 IAU

Alan Penny, Pawel Artymowicz, Anne-Marie Lagrange, \& Sara Russell, eds.

\title{
Spectral energy distributions and structures of debris discs
}

\author{
W.R.F. Dent and M.C. Wyatt \\ UK ATC, Royal Observatory, Blackford Hill, Edinburgh EH9 3HJ, UK
}

W.S. Holland, J.S. Greaves, and I.M. Coulson

Joint Astronomy Centre, 660 N. Aohoku Pl., Hilo, HI 96720, USA

D.M. Walther

Gemini Observatory, Hilo, HI 96720, USA

\begin{abstract}
New photometry of main-sequence debris discs has been carried out at 850 and $450 \mu \mathrm{m}$; the derived SEDs indicate that the dust can lie in either thin rings or radially-extended discs, as seen directly in the few nearby objects which are resolvable. All such objects are consistent with a long wavelength opacity index $\beta$ of $1.0 \pm 0.2$ - similar to T Tauri stars, but significantly lower than embedded objects.
\end{abstract}

\section{Introduction}

The link between the dust found around main sequence stars and pre main sequence objects is not fully understood. Grains around the former are thought to be part of a collisional cascade from larger bodies (eg Backman \& Paresce, 1993), while in the latter case they are believed to be still undergoing growth (eg Schmitt, Henning, \& Mucha, 1997). However, many Vega-excess stars are relatively young, which suggests that there may be a connection.

Images of the material around main-sequence stars shows the dust lies in rings or more extended discs. Often ascribed to the influence of planets, the observed structure should reveal something about the prevalence of massive bodies at 10-100 AU from the central stars (eg Liou \& Zook, 1999). But currently only $\sim 10$ relatively nearby objects have well-resolved images, and so the only way to study most of them is through their Spectral Energy Distributions.

It is clearly difficult to derive unique solutions to SEDs, as has been amply demonstrated in the case of young stars. Derived temperatures and $\beta$ are often degenerate, particularly when the shape of the SED is ill-defined due to gaps in wavelength coverage, or when only a restricted wavelength range and low signal:noise is available (Dent et al. 1998). The current observations are based on new high s:n photometry at 850 and $450 \mu \mathrm{m}$ with SCUBA on the JCMT, and are combined with published results to derive the SEDs. 

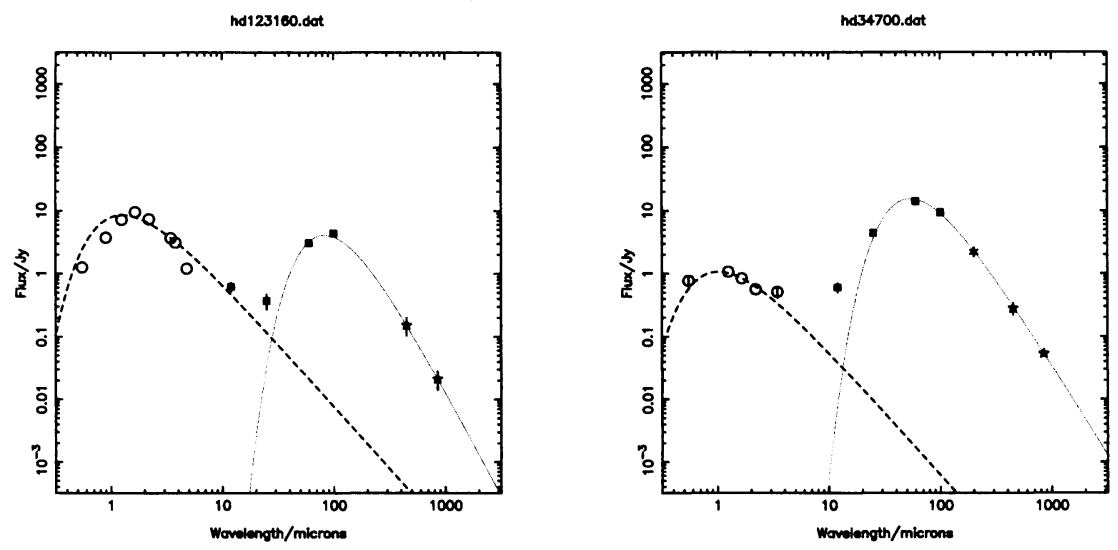

Figure 1. SEDs of the Type II objects HD123160 and HD34700. In this and Fig 2, the stellar photosphere (dashed line) is approximated by a black body, and the fit to the excess shown dotted. Additional data is mostly from the IRAS PSC, and Sylvester et al. (1996).

\section{Results}

SEDs for four of the targets are shown in Figs $1 \& 2$. For convenience, we divide the SED characteristics into three types. In the archetypical stars such as $\epsilon$ Eri and Fomalhaut (designated Type I), a single-temperature modified black-body adequately fits the excess (eg Dent et al. 2000). Type II objects (Fig 1) have an additional weak mid-IR component, which dominates in Type III objects (Fig 2). In some cases (eg HD139614), the latter may also have a near-IR excess. Coulson, Walther, \& Dent (1998) also noted several more of these Type III objects, which also tend to have higher total excesses (as measured by $\tau$, the ratio of excess to stellar luminosity). Rather than two or three components, a better fit may actually come from a spread of dust temperatures in Type III objects.

The addition of accurate photometry in the current dataset, particularly at wavelengths intermediate between previous mm-wave and IRAS measurements, provides less interdependency in the derivations of $\beta$ and T. Table 1 shows a summary of the results, which compares the main-sequence objects with optically-visible T Tauri stars and embedded YSOs (from Dent et al. 1998).

Table 1. Comparison of temperature and $\beta$ of coolest dust component for main-sequence Vega-excess stars and younger objects.

\begin{tabular}{lllc}
\hline Object & T $(\mathrm{K})$ & $\beta$ & No. of objects \\
\hline Type I m-s & $35-80$ & $0.9 \pm 0.2$ & 3 \\
Type II m-s & $40-85$ & $1.1 \pm 0.2$ & 4 \\
Type III m-s & $25-40(80-150)$ & $1.0 \pm 0.2$ & 4 \\
Optical T Tauri & $30-60$ & $0.0-1.0$ & $10+$ \\
Embedded YSOs & $30 \pm 10$ & $1.5 \pm 0.5$ & $35+$ \\
\hline \hline
\end{tabular}



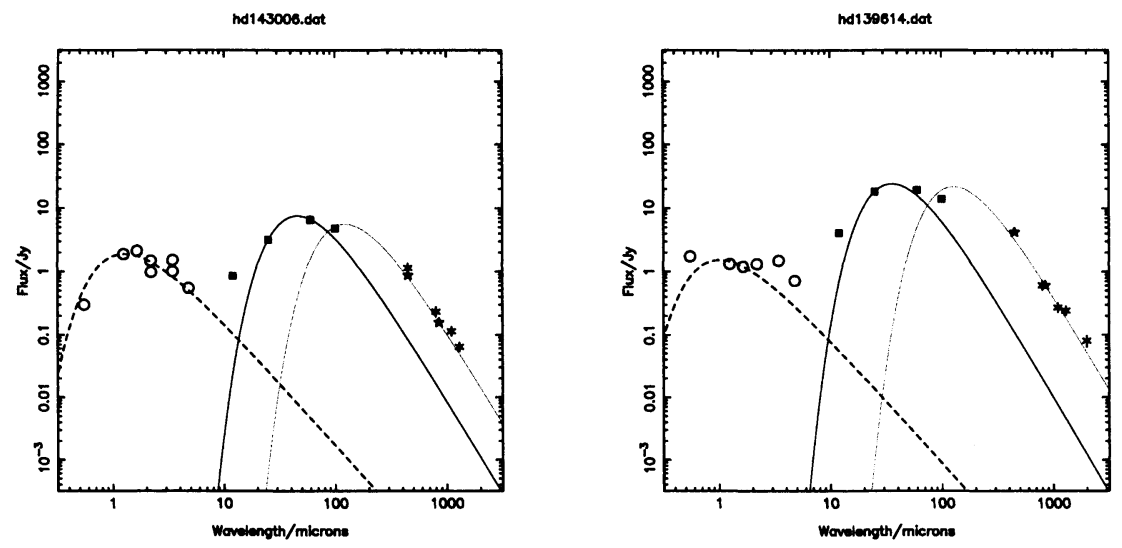

Figure 2. SEDs of the Type III objects HD143006 and HD139614. Note the extra component needed to account for mid and near-IR.

\section{Discussion}

The different spreads of dust temperature suggest that dust either lies in narrow isothermal rings of radius typically $\sim 80$ AU (Type I), or in broad radiallyextended discs (Type III). In a few cases this can be confirmed through imaging (eg Fomalhaut \& $\beta$ Pic). Thin ring structures may result from disturbing planets, suggesting that these SED differences may be due to the presence or absence of such planets in the region 20-100 AU from these main-sequence stars.

Table 1 shows no clear dependence of temperature on the SED type of mainsequence stars; furthermore all have $\beta \sim 1.0 \pm 0.2$ - similar to optically-visible $\mathrm{T}$ Tauri stars. In these objects, this is thought to arise from large "fluffy" grains. However, there is a clear difference between this dust and that around embedded YSOs. The conclusion is that debris disc dust is clearly different from YSO dust, but may be similar in composition, shape and even origin to $\mathrm{T}$ Tauri dust.

\section{References}

Backman, D.E., \& Paresce, F. 1993, in Protostars and Planets III, ed. E.H. Levy \& J.I. Lunine (Univ. of Arizona), 1253

Coulson, I.M., Walther, D.M., \& Dent, W.R.F. 1998, MNRAS, 296, 934

Dent, W.R.F., Matthews, H.E., Ward-Thompson, D.W. 1998, MNRAS, 301, 1049

Dent, W.R.F., Walker, H.J., Holland, W.S. \& Greaves, J.S. 2000, MNRAS, 314, 702

Liou, J.-C. \& Zook, H.A. 1999, AJ, 118, 580

Schmitt, W., Henning, T., \& Mucha, R. 1997, A\&A, 325, 569

Sylvester, R.J., Skinner, C.J., Barlow, M.J. \& Mannings, V. 1996, MNRAS, 279, 915 